\title{
Resonator-Inspired Metamaterial Sensor: Design and Experimental Validation for Measuring Thickness of Multi-Layered Structures
}

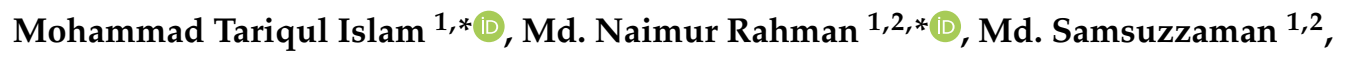 \\ Mohd Fais Mansor ${ }^{1}$ (D) and Norbahiah Misran ${ }^{1}$ \\ 1 Centre of Advanced Electronic and Communication Engineering, Universiti Kebangsaan Malaysia, \\ UKM Bangi, Selangor 43600, Malaysia; samsuzzaman@ukm.edu.my (M.S.); \\ m.mansor@ukm.edu.my (M.F.M.); bahiah@ukm.edu.my (N.M.) \\ 2 Patuakhali Science and Technology University, Patuakhali 8602, Bangladesh \\ * Correspondence: tariqul@ukm.edu.my (M.T.I.); p86256@siswa.ukm.edu.my (M.N.R.); \\ Tel.: +60-193-666192 (M.T.I.)
}

Received: 1 August 2018; Accepted: 16 October 2018; Published: 1 December 2018

\begin{abstract}
A digit 8-shaped resonator inspired metamaterial is proposed herein for sensor applications. The resonator is surrounded by a ground frame and excited by a microstrip feedline. The measurement of the sensor can be performed using common laboratory facilities in lieu of using the waveguide, as the resonator, ground frame, and feedline are all on the same microstrip. To achieve metamaterial properties, more than one unit cell is usually utilized, whereas, in this work, a single cell was used to achieve the metamaterial characteristics. The properties of the metamaterial were investigated to find the relationship between the simulation and measurements. The proposed metamaterial sensor shows considerable sensitivity in sensor application. For the sensor application, FR4 and Rogers RO4350 materials were used as the over-layer. The sensor can measure dielectric thickness with a sensitivity of $625 \mathrm{MHz} / \mathrm{mm}, 468 \mathrm{MHz} / \mathrm{mm}$, and $354 \mathrm{MHz} / \mathrm{mm}$ for the single over-layer, double over-layers, and multiple over-layers, respectively. The proposed prototype can be utilized in several applications where metamaterial characteristics are required.
\end{abstract}

Keywords: metamaterial sensor; resonator; thickness; sensitivity

\section{Introduction}

Since their first introduction in 1968, metamaterials attracted researchers working with the characterization of metamaterials. Researchers introduced several resonator-based metamaterials which work in different frequencies [1]. For ultra-wideband microwave imaging, a split-ring resonator (SRR)-inspired metamaterial antenna was utilized [2]. Terahertz sensing was described using an SRR-based metamaterial [3]. Again, a terahertz-sensing device was introduced in Reference [4] using a broadside-coupled triangular SRR-based metamaterial. A microwave metamaterial sensor was introduced where transmission lines worked as the building block [5]. A complementary SRR was used to characterize the dielectric properties of a metamaterial operating in the $0.8-1.3 \mathrm{GHz}$ band [6]. Dielectric characterization of the microfluidics was done with a metamaterial sensor using a microstrip-coupled complementary split-ring resonator (CSRR) [7]. An H-shaped resonator-based metamaterial was developed and experimented in sensor and absorber applications [8]. To find out the resonant frequencies and Q-factors of whispering-gallery modes (WGMs), a microring resonator-based metamaterial sensor was introduced [9]. For the calculation of resonant frequencies and the electric field distribution of WGMs, a negative permeability metamaterial sensor was developed and described in Reference [10]. A metamaterial-based wireless sensor was used to measure strain and displacement 
during the deformation of elastic and plastic materials, which is beneficial in health-monitoring systems [11]. An S-shaped split-ring resonator (SSRR)-based metamaterial sensor, using a coplanar waveguide (CPW) transmission line, was used to find the angular velocity and displacement [12]. An SRR-inspired metamaterial-based microfluidic sensor was utilized to identify, classify, and characterize biochemical and chemical analyses [13]. A CSRR-based sensor was used to measure the thickness and permittivity of a multi-layered structure using the non-invasive microwave method [14]. A quasi-static resonator was used to measure the non-destructive thickness, which also used a CSRR operating between 1.60 to $2.30 \mathrm{GHz}$ bands [15]. A planar resonator sensor was utilized to monitor the solid element in a lossy medium operating at $1.50 \mathrm{GHz}$ having a quality factor of 230 [16]. A metamaterial-based microwave sensor was introduced to measure the impurities in a liquid based on the transmission quality of the sensor [17]. A resonator-based microwave sensor was used to monitor the sensitivity enhancement for $\mathrm{CO}_{2}$ detection [18]. A differential sensor based on two SRRs was utilized to characterize solid dielectric samples [19]. A microwave gas sensor was represented using electromagnetic transducers to monitor volatile organic particles [20]. A wireless biosensor was rendered for the detection of bacteria in milk using a capacitor-inductance tank [21]. A microstrip gas sensor based on a ring resonator was introduced, and was used to detect humidity and ammonia [22]. A substrate-integrated waveguide sensor was presented as a chemical sensor, using ethanol and deionized water as dielectric fluids [23]. Most of the reviewed sensors are large in size and complex to design. Again, some sensors stated above have limited sensitivity.

In this article, a resonator-inspired metamaterial sensor is proposed based on microstrip technology. Simulated and experimented metamaterial properties were investigated extensively, and both results matched quite well. Finally, the metamaterial sensor was utilized to identify unknown materials of different thickness. The proposed sensor has better sensitivity than the earlier reported similar works.

\section{Model, Prototype, and Experiment}

The metamaterial consists of a digit 8-shaped resonator, surrounded by a ground frame, along with a feeding transmission line (FTL) exhibiting metamaterial characteristics. The metamaterial was prototyped on the FR4 material, which has 4.6 relative permittivity and 0.02 loss tangent. The resonator and the ground frame were sketched on the same portion of the substrate. The feeding transmission line (FTL) was sketched on the opposite portion of the substrate. The excitation is supplied to the resonator through the FTL. The measurement of the sensor can be performed using common laboratory facilities in lieu of using the waveguide, as the resonator, ground frame, and feedline are all on the same microstrip. To achieve metamaterial properties, more than one unit cell is usually utilized, whereas, in this work, a single cell was used to achieve the metamaterial characteristics. The design of the resonator is simpler compared to the previously reported planar resonator-based sensors. Again, the previously reported similar works used larger resonator sensors to detect the over-layers. Moreover, some sensors have limited sensitivity as compared to the proposed sensor. The metamaterial was designed and investigated using the High-Frequency Structural Simulator (HFSS) software. The geometry of the sensor is shown in Figure 1. Figure 2 depicts the prototype of the digit 8-shaped resonator-inspired metamaterial sensor. The N5227A PNA Microwave Network Analyzer (Agilent Technologies, Santa Clara, CA, USA) $(10 \mathrm{MHz}-67 \mathrm{GHz})$ has been used to measure the sensor. The reflection and transmission coefficients of the sensor are depicted in Figure 3, where resonances were found at $3 \mathrm{GHz}$ and $6.5 \mathrm{GHz}$. The transmission resonant frequency was observed at $6.0 \mathrm{GHz}$. 


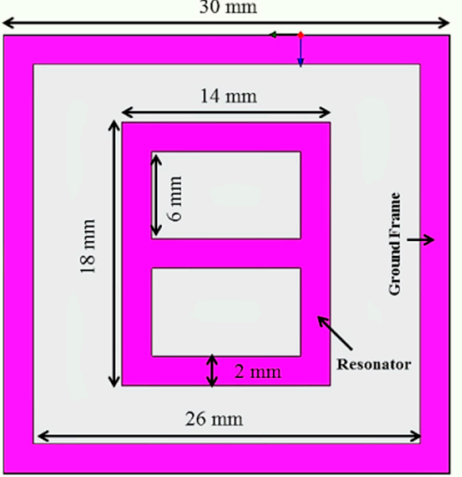

(a)

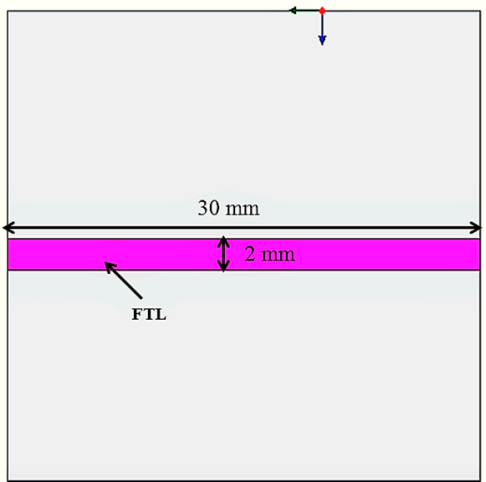

(b)

Figure 1. Geometry of the sensor: (a) top side, and (b) bottom side.

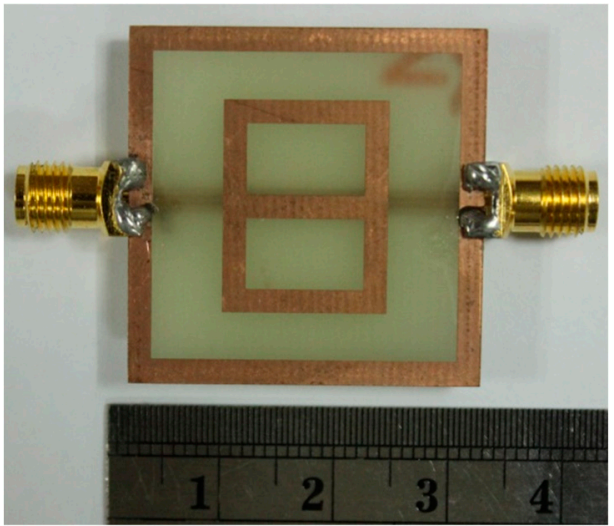

(a)

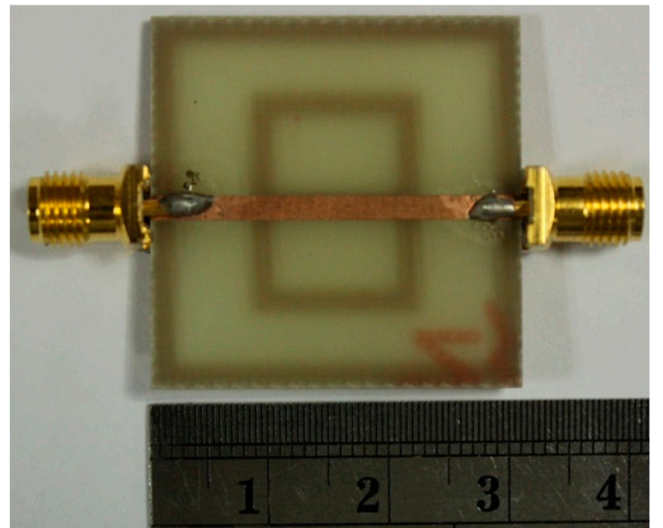

(b)

Figure 2. A prototype of the sensor: (a) top side, and (b) bottom side.

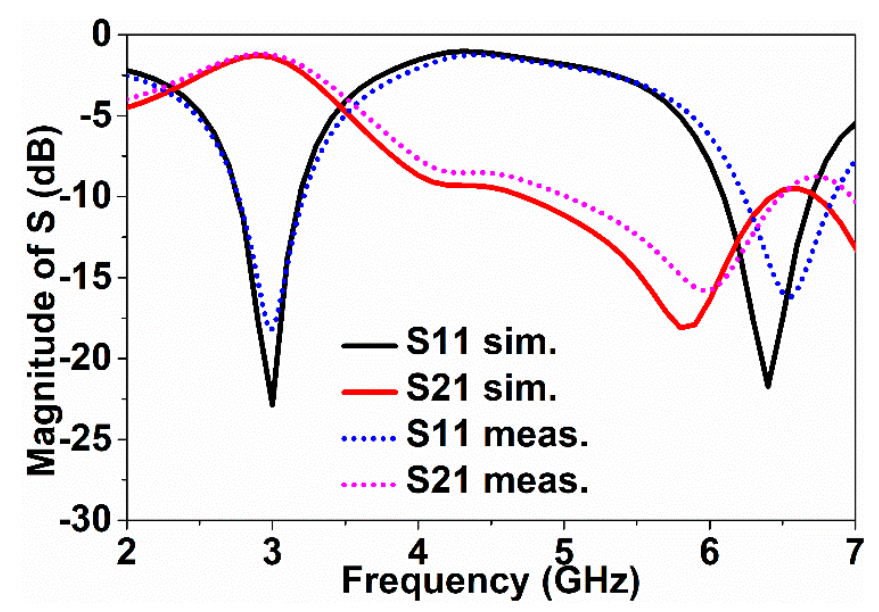

Figure 3. The magnitude of the S-parameter.

\section{Characterization of Metamaterial}

The Nicolson-Ross-Weir (NRW) method was used to characterize the metamaterial properties. The values of the refractive index depend on the values of the permeability and permittivity. The NRW method is mathematically expressed by the subsequent equations [1].

$$
\Gamma=\left[\left(S_{11}^{2}+S_{21}^{2}+1\right) 2 S_{11}\right] \pm \sqrt{\left[\left(S_{11}^{2}+S_{21}^{2}+1\right) 2 S_{11}\right]^{2}-1}
$$




$$
\begin{gathered}
T=\frac{\left(S_{11}+S_{21}-\Gamma\right)}{\left[1-\Gamma\left(S_{11}+S_{21}\right)\right]} \\
\kappa=\left[\log \left(\frac{1}{|T|}\right)\right]+j[2 m \pi-\operatorname{phase}(T)] / d, m=0, \pm 1, \pm 2, \pm 3, \\
\varepsilon=\frac{\kappa}{\kappa_{0}}\left(\frac{1-\Gamma}{1+\Gamma}\right), \\
\mu=\frac{\kappa}{\kappa_{0}}\left(\frac{1+\Gamma}{1-\Gamma}\right),
\end{gathered}
$$

where $\mu$ denotes permeability, $\varepsilon$ denotes permittivity, and $\kappa_{0}$ denotes the propagation constant in free space. Using these values of permeability and permittivity, the effective constituents were calculated, following the Drude model dispersion.

At the peak of the transmission $\left(S_{21}\right)$, the effective permittivity and permeability became negative. In Figure $4 \mathrm{a}$, the effective permittivity remains negative from $2.7 \mathrm{GHz}$ to $4.1 \mathrm{GHz}$, whereas Figure $4 \mathrm{~b}$ depicts a negative permeability from $2 \mathrm{GHz}$ to $6 \mathrm{GHz}$. The refractive index became negative as the permittivity and permeability remained negative, as shown in Figure 4c. Therefore, the metamaterial depicted double-negative properties in the bandwidth from $2.7 \mathrm{GHz}$ to $4.1 \mathrm{GHz}$, and all parameters had a negative peak at $3 \mathrm{GHz}[1,24]$.

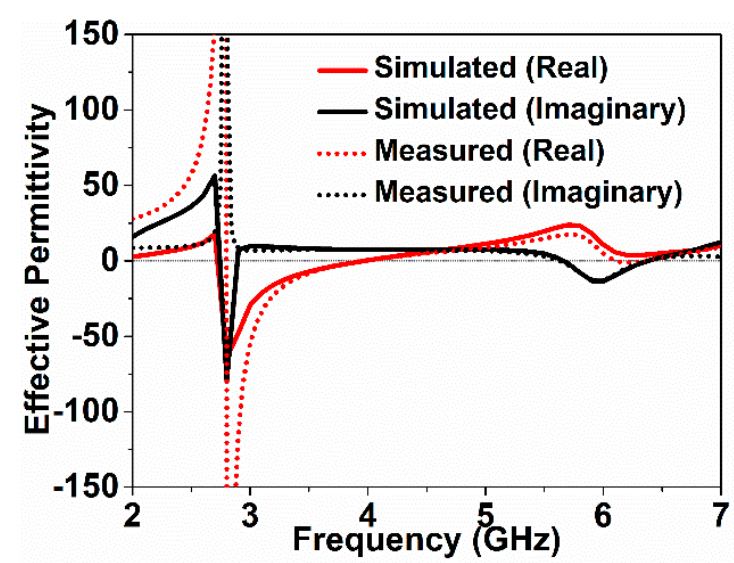

(a)

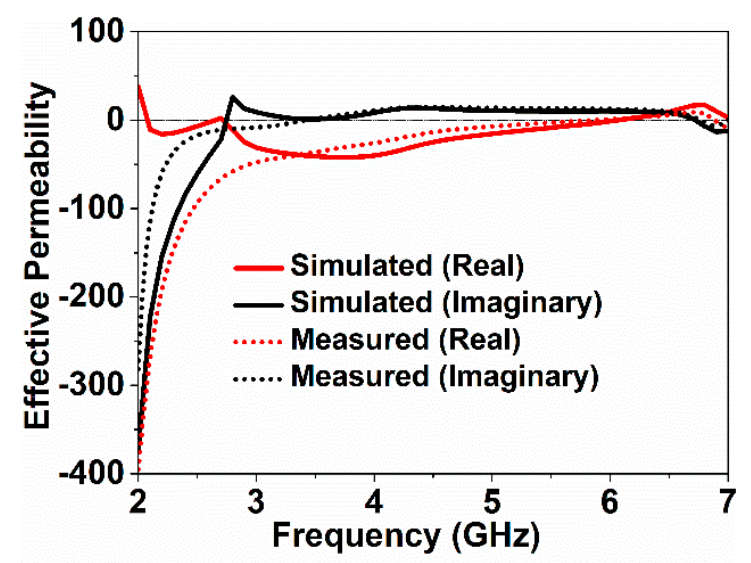

(b)

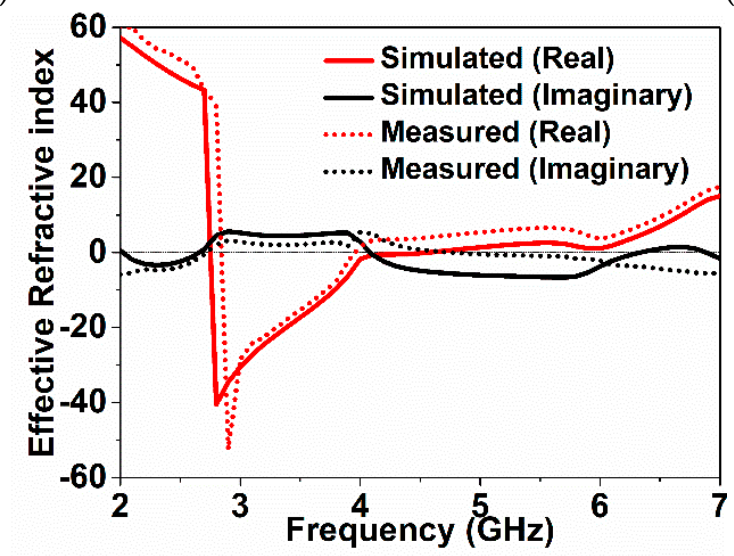

(c)

Figure 4. Effective parameters of the sensor: (a) permittivity, (b) permeability, and (c) refractive index.

To describe the resonance type, the surface current distribution of the sensor was included. Figure 5 represents the surface current distribution of the sensor. 


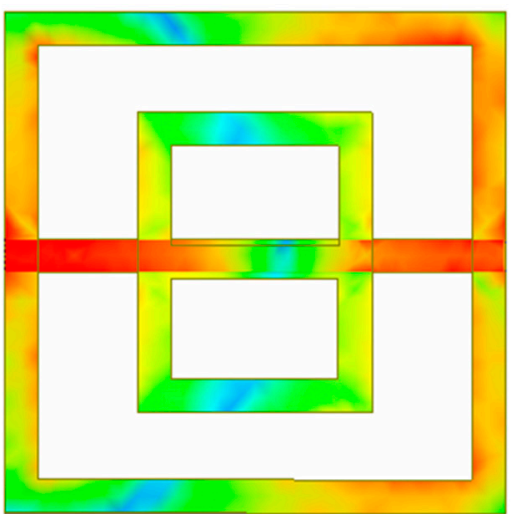

(a)

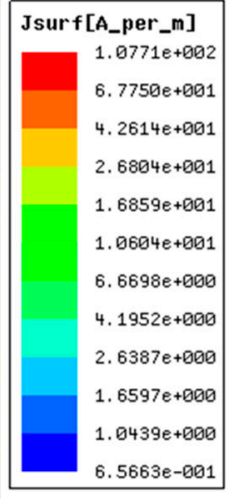

$0439 e+000$

$5663 e-001$

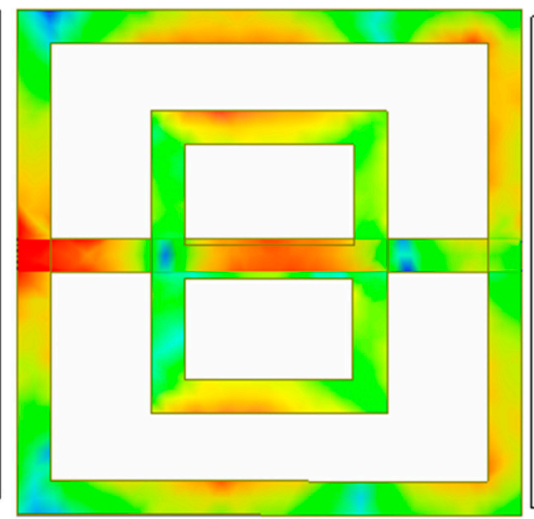

(b)

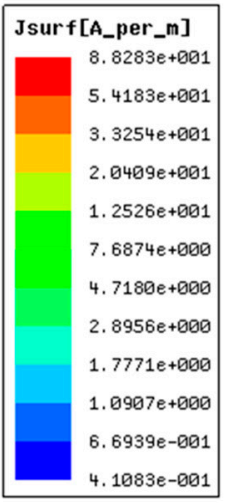

Figure 5. Current distribution of the sensor: (a) at $3.0 \mathrm{GHz}$, and (b) at $6.5 \mathrm{GHz}$.

The current was distributed mainly on the resonator and the FTL. Two opposite directional magnetic dipoles were formed on the ground frame due to the symmetrical rotation of the surface currents on the resonator. Figure 6 represents the magnetic field distribution of the sensor.

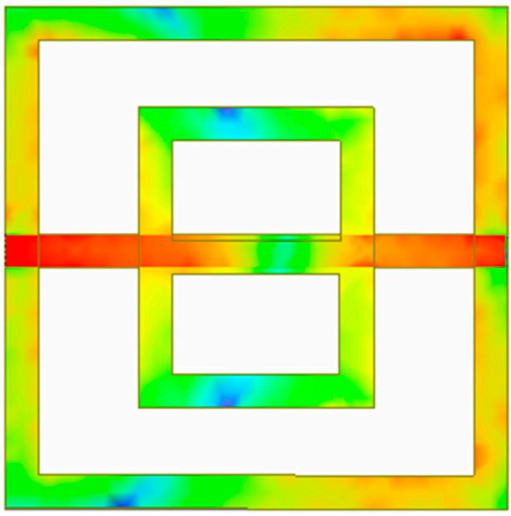

(a)

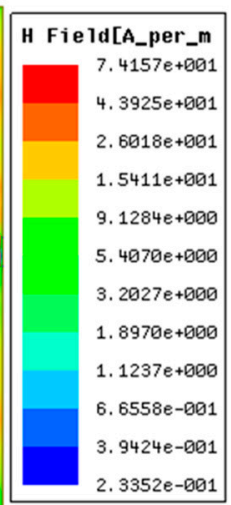

$3352 e-001$
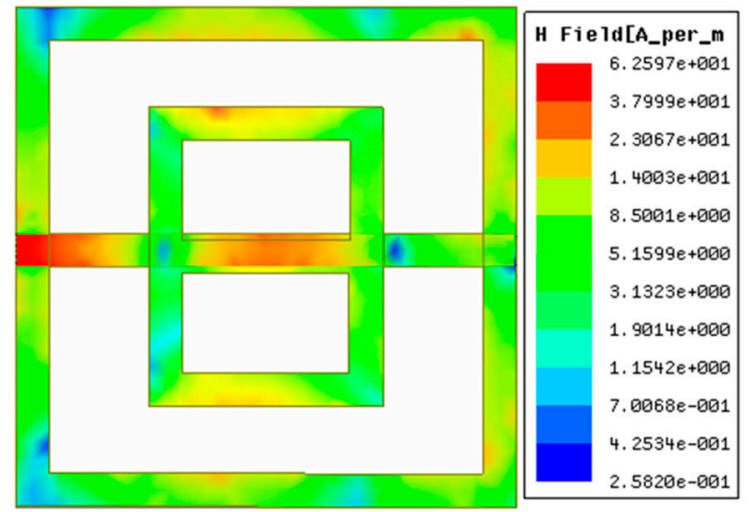

(b)

Figure 6. Magnetic field distribution of the sensor: (a) at $3.0 \mathrm{GHz}$, and (b) at $6.5 \mathrm{GHz}$.

The FTL was mutually attached to the ground and the resonator with its electric field. Hence, the sensor presents inductive and capacitive behavior because of the ground frame and the gap between the ground and the resonator. Diamagnetism appears due to the induced magnetic field, which is opposite to the external magnetic field. For the diamagnetism, the sensor shows negative permeability. Moreover, the ground frame creates negative permittivity and negative permeability through the inductive-capacitive properties of the sensor.

\section{Metamaterial as Sensor}

The resonator-inspired metamaterial sensor was used in a double-sided sensor application. Unknown objects were detected, upon being used as the over-layer. The sensor was experimented using different over-layers with different thickness by means of the change in frequency responses. The sensitivity of the sensor was determined considering the change in dielectric thickness with the permittivity kept constant.

The frequency responses for changes in over-layer thickness were observed. In the simulation, an over-layer having 4.4 dielectric constants was used, as it has parameter values close to the FR4 substrate. The simulated frequency responses with the changes in over-layer thickness are shown in Figure 7. The resonance shifted downward when over-layer thickness increased. The metamaterial sensor with the over-layer constitutes a resistor/inductor/capacitor (RLC) circuit, 
where the resonance is determined by effective capacitance and inductance. As effective inductance and capacitance vary when the over-layer thickness increases due to the self-resonance, the resonance shifts downward because of the resonance frequency changes with the effective capacitance and inductance. As the ground plane around the resonator provides additional capacitance and inductance, it has significant effects on the resonance frequency.

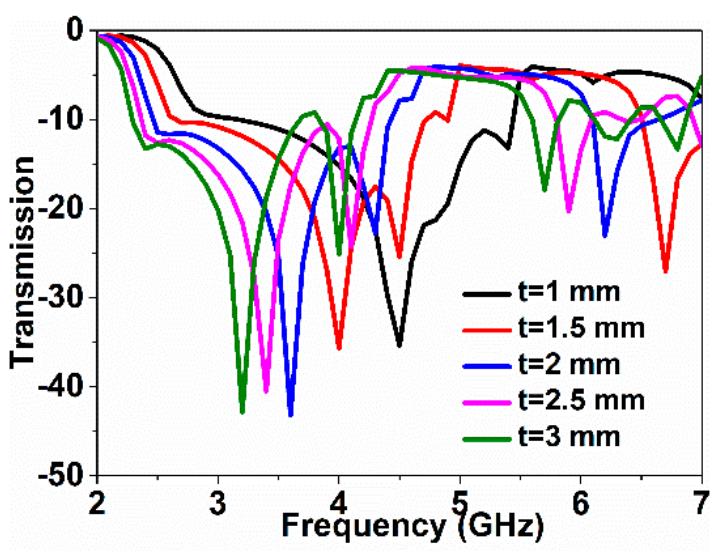

Figure 7. Change in transmission with the change in over-layer thickness.

In the measurement, the FR4 substrate, with $1.66 \mathrm{~mm}$ thickness with 4.6 relative permittivity, and the Rogers RO4350 substrate, with $1.53 \mathrm{~mm}$ thickness with 3.66 relative permittivity, were utilized as the over-layers. Figure 8 depicts the sensor with over-layers along with the PNA measurement set-up. Figure 9 depicts the simulated and experimental resonance responses of the metamaterial sensor when detecting the unknown material.

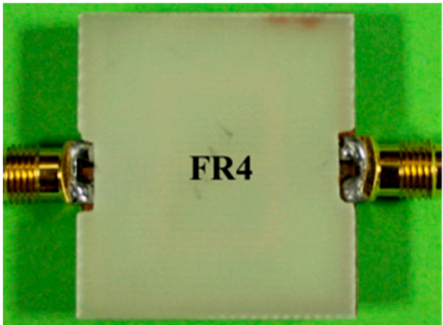

(a)

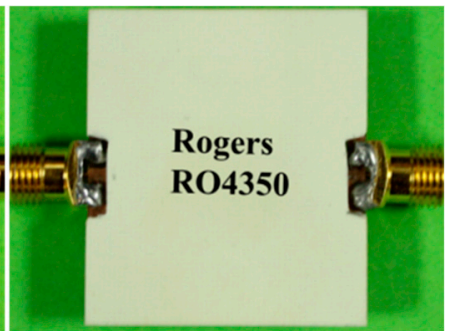

(b)

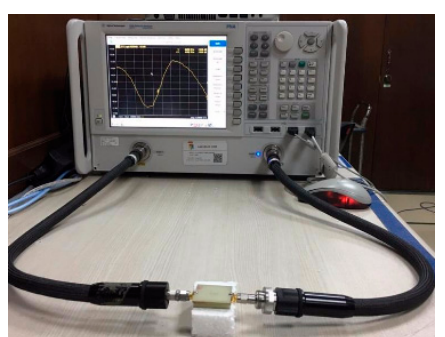

(c)

Figure 8. Sensors with over-layers: (a) FR4 over-layer, and (b) Rogers RO4350 over-layer. (c) PNA measurement set-up.

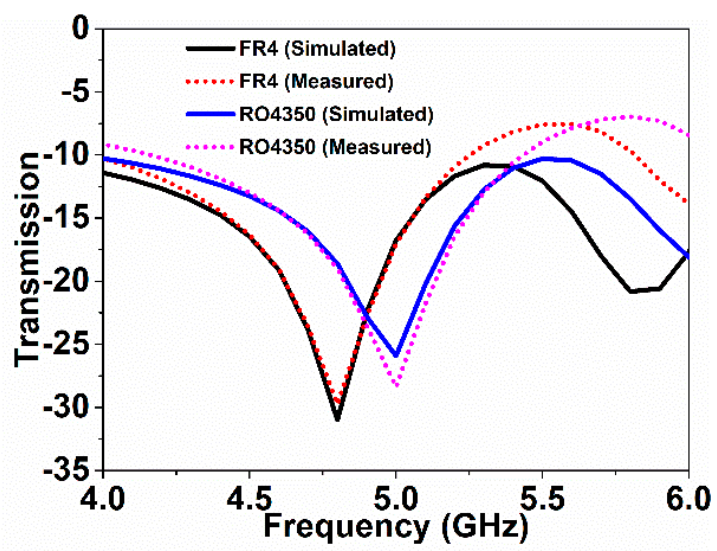

Figure 9. Transmission responses with FR4 and Rogers RO4350 over-layers. 
The frequency shifts depend on the over-layer thickness and the over-layer's permittivity. The larger the values of thickness and permittivity are, the more the resonance shifts downward. The higher the over-layer thickness and permittivity become, the larger the downshift of the resonance becomes. In this article, the sensitivity of the sensor was measured considering the change in over-layer thickness upon keeping permittivity constant. In Figure 9, the resonance moved downward when the over-layer thickness increased. The over-layer thickness was increased by adding etched substrates, making a stack of substrates. There existed negligible gaps between the stack of substrates. However, the measured results differed in some cases due to the little gaps between the stacked substrates. The thickness of FR4 is larger than that of the Rogers RO4350 substrate; thus, for the FR4 over-layer, the resonance decreases further than the Rogers RO4350 over-layer. For specific clarification, the transmission resonance was at $6.0 \mathrm{GHz}$ with no over-layer; however, using the Rogers RO4350 and FR4 over-layers, the resonances moved to $5.0 \mathrm{GHz}$ and $4.8 \mathrm{GHz}$, respectively. As the thickness increased, the resonance frequency shifted downward. The greater the thickness of the over-layer became, the more the resonance shifts occurred. Using the shifting values of the resonance frequency, the sensitivity of the sensor was calculated. Considering the frequency shift as a function of over-layer thickness, the sensitivity of the metamaterial sensor was $625 \mathrm{MHz} / \mathrm{mm}$ for a single over-layer. For a double over-layer, FR4 + FR4 and Rogers RO4350 + Rogers RO4350 substrates were utilized. Figure 10 depicts the resonance responses for the double over-layers.

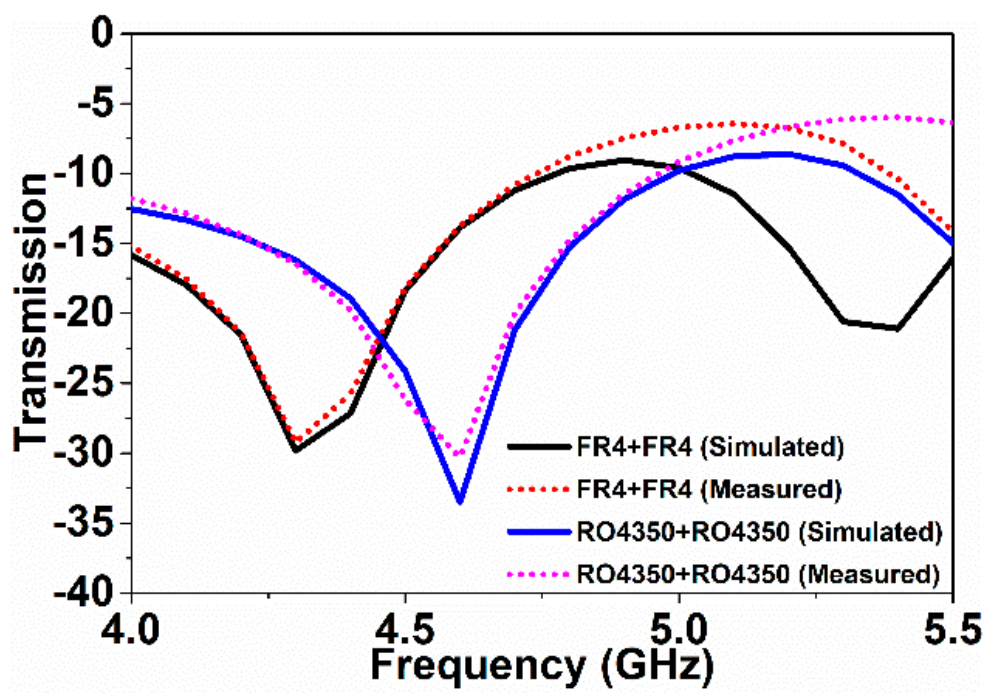

Figure 10. Transmission variances with FR4 and Rogers RO4350 double over-layers.

The resonance moved downward when the thickness of the over-layer increased. Hence, for the Rogers RO4350 + Rogers RO4350 and FR4 + FR4 double over-layers, the resonances moved to $4.6 \mathrm{GHz}$ and $4.3 \mathrm{GHz}$, respectively. Considering the frequency shift as a function of over-layer thickness, the sensitivity of the metamaterial sensor was $468 \mathrm{MHz} / \mathrm{mm}$ for the double over-layer.

For a further extension of the experiment, multiple over-layers were considered. In this case, FR4 + FR4 + FR4 and Rogers RO4350 + Rogers RO4350 + Rogers RO4350 substrates were utilized. The simulated and experimented results are plotted in Figure 11. The resonance downshifted when the thickness increased. Using the Rogers RO4350 + Rogers RO4350 + Rogers RO4350 and FR4 + FR4 + FR4 multiple over-layers, the resonances downshifted to $4.4 \mathrm{GHz}$ and $4.1 \mathrm{GHz}$, respectively. Considering the frequency shift as a function of over-layer thickness, the sensitivity of the metamaterial sensor was $354 \mathrm{MHz} / \mathrm{mm}$ for the multiple over-layers experimented above. 


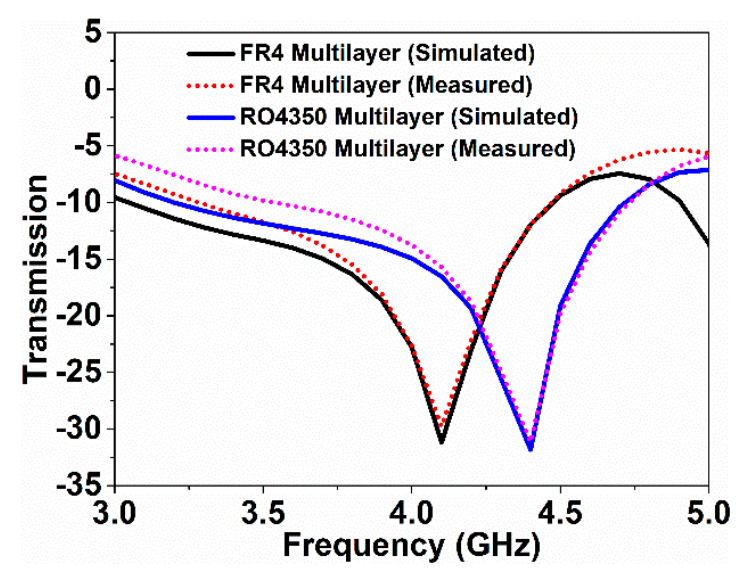

Figure 11. Transmission variances with FR4 and Rogers RO4350 multiple over-layers.

For the over-layer, double over-layer, and multiple over-layer variants, the simulated and measured values agreed well. On the basis of the simulations and measurements, it can be concluded that the metamaterial sensor can measure the dielectric thickness of multi-layered structures with considerable sensitivity.

The proposed sensor outperformed previous related works according to sensor size, sensor type, and sensitivity. Table 1 shows the comparative characteristics between the proposed sensor and the previous related sensors.

Table 1. Comparative characteristics of the proposed sensor with previous related sensors. SRR—split-ring resonator; SSRR—S-shaped SSR; TSRR—triangular SRR; CSRR—complementary SRR.

\begin{tabular}{cccccc}
\hline Reference & Sensor Size & Resonator & Operating Band & Sensing Base & Sensitivity \\
\hline$[1]$ & $35 \mathrm{~mm} \times 35 \mathrm{~mm}$ & S-shaped & $\mathrm{GHz}$ & Frequency shift & Not reported \\
{$[3]$} & $87.5 \mu \mathrm{m} \times 87.5 \mu \mathrm{m}$ & SSRR & $\mathrm{THz}$ & Frequency shift & Not reported \\
{$[4]$} & $75 \mu \mathrm{m} \times 75 \mu \mathrm{m}$ & TSRR & $\mathrm{THz}$ & Frequency shift & Not reported \\
{$[7]$} & Not reported & CSRR & $\mathrm{GHz}$ & Frequency shift & $400 \mathrm{MHz}$ \\
{$[19]$} & Not reported & SRR & $\mathrm{GHz}$ & Frequency shift & $72 \mathrm{MHz}$ \\
Proposed & $30 \mathrm{~mm} \times 30 \mathrm{~mm}$ & 8-shaped & $\mathrm{GHz}$ & Frequency shift & Max $625 \mathrm{MHz}$ \\
\hline
\end{tabular}

\section{Conclusions}

A digit-8 shaped resonator-inspired metamaterial sensor was modeled, prototyped, and analyzed herein. The sensor was experimented using usual laboratory apparatus in lieu of waveguides. Both the simulated and measured values matched well, showing the double-negative metamaterial characteristics of the metamaterial sensor in the operating region. The proposed sensor measured the over-layer thickness, using FR4 and Rogers RO4350 substrates as the over-layers. The sensor was used to measure the dielectric thickness of multi-layered structures with considerable sensitivities of $625 \mathrm{MHz} / \mathrm{mm}, 468 \mathrm{MHz} / \mathrm{mm}$, and $354 \mathrm{MHz} / \mathrm{mm}$ for the single over-layer, double over-layers, and multiple over-layers, respectively.

Author Contributions: M.N.R. and M.S. made substantial contributions to conception, design, and analysis. M.T.I., M.F.M., and N.M. provided the necessary instructions for experimental and writing purposes.

Funding: The authors would like to thank the Centre of Advanced Electronic and Communication Engineering, Universiti Kebangsaan Malaysia (UKM) for the financial support to conduct this research Grant No. AP-2017-007/1 and MI-2017-001.

Acknowledgments: The authors acknowledge the PCB lab and Microwave Lab of the dept. of Electrical, Electrical and System Engineering, Universiti Kebangsaan Malaysia (UKM), Malaysia for using the fabrication and measurement facilities.

Conflicts of Interest: The authors declare no conflict of interest. 


\section{References}

1. Sabah, C.; Nesimoglu, T. Design and characterization of a resonator-based metamaterial and its sensor application using microstrip technology. Opt. Eng. 2016, 55, 027107. [CrossRef]

2. Islam, M.M.; Islam, M.T.; Samsuzzaman, M.; Faruque, M.R.I.; Misran, N.; Mansor, M.F. A miniaturized antenna with negative index metamaterial based on modified SRR and CLS unit cell for UWB microwave imaging applications. Materials 2015, 8, 392-407. [CrossRef] [PubMed]

3. Sabah, C.; Roskos, H. Terahertz sensing application by using planar split-ring-resonator structures. Microsyst. Technol. 2012, 18, 2071-2076. [CrossRef]

4. Sabah, C.; Roskos, H.G. Broadside-coupled triangular split-ring-resonators for terahertz sensing. Eur. Phys. J. Appl. Phys. 2013, 61, 30402. [CrossRef]

5. Schueler, M.; Mandel, C.; Puentes, M.; Jakoby, R. Metamaterial inspired microwave sensors. IEEE Microw. Mag. 2012, 13, 57-68. [CrossRef]

6. Boybay, M.S.; Ramahi, O.M. Material characterization using complementary split-ring resonators. IEEE Trans. Instrum. Meas. 2012, 61, 3039-3046. [CrossRef]

7. Ebrahimi, A.; Withayachumnankul, W.; Al-Sarawi, S.; Abbott, D. High-sensitivity metamaterial-inspired sensor for microfluidic dielectric characterization. IEEE Sens. J. 2014, 14, 1345-1351. [CrossRef]

8. Sabah, C.; Taygur, M.M.; Zoral, E.Y. Investigation of microwave metamaterial based on H-shaped resonator in a waveguide configuration and its sensor and absorber applications. J. Electromagn. Waves Appl. 2015, 29, 819-831. [CrossRef]

9. Huang, M.; Yang, J.; Jun, S.; Mu, S.; Lan, Y. Simulation and analysis of a metamaterial sensor based on a microring resonator. Sensors 2011, 11, 5886-5899. [CrossRef] [PubMed]

10. Sun, J.; Huang, M.; Yang, J.-J.; Li, T.-H.; Lan, Y.-Z. A microring resonator based negative permeability metamaterial sensor. Sensors 2011, 11, 8060-8071. [CrossRef] [PubMed]

11. Ozbey, B.; Demir, H.V.; Kurc, O.; Erturk, V.B.; Altintas, A. Wireless measurement of elastic and plastic deformation by a metamaterial-based sensor. Sensors 2014, 14, 19609-19621. [CrossRef] [PubMed]

12. Naqui, J.; Coromina, J.; Karami-Horestani, A.; Fumeaux, C.; Martín, F. Angular displacement and velocity sensors based on coplanar waveguides (CPWs) loaded with S-shaped split ring resonators (S-SRR). Sensors 2015, 15, 9628-9650. [CrossRef] [PubMed]

13. Withayachumnankul, W.; Jaruwongrungsee, K.; Tuantranont, A.; Fumeaux, C.; Abbott, D. Metamaterial-based microfluidic sensor for dielectric characterization. Sens. Actuators A Phys. 2013, 189, 233-237. [CrossRef]

14. Lee, C.-S.; Yang, C.-L. Thickness and permittivity measurement in multi-layered dielectric structures using complementary split-ring resonators. IEEE Sens. J. 2014, 14, 695-700. [CrossRef]

15. Boybay, M.S.; Ramahi, O.M. Non-destructive thickness measurement using quasi-static resonators. IEEE Microw. Wirel. Compon. Lett. 2013, 23, 217-219. [CrossRef]

16. Zarifi, M.H.; Daneshmand, M. Monitoring Solid Particle Deposition in Lossy Medium Using Planar Resonator Sensor. IEEE Sens. J. 2017, 17, 7981-7989. [CrossRef]

17. Soffiatti, A.; Max, Y.; Silva, S.G.; de Mendonça, L.M. Microwave Metamaterial-Based Sensor for Dielectric Characterization of Liquids. Sensors 2018, 18, 1513. [CrossRef] [PubMed]

18. Zarifi, M.H.; Gholidoust, A.; Abdolrazzaghi, M.; Shariaty, P.; Hashisho, Z.; Daneshmand, M. Sensitivity enhancement in planar microwave active-resonator using metal organic framework for $\mathrm{CO}_{2}$ detection. Sens. Actuators B Chem. 2018, 255, 1561-1568. [CrossRef]

19. Ebrahimi, A.; Scott, J.; Ghorbani, K. Differential Sensors Using Microstrip Lines Loaded with Two Split Ring Resonators. IEEE Sens. J. 2018, 18, 5786-5793. [CrossRef]

20. Bahoumina, P.; Hallil-Abbas, H.; Lachaud, J.-L.; Rebiere, D.; Dejous, C.; Abdelghani, A.; Frigui, K.; Bila, S.; Baillargeat, D.; Zhang, Q. VOCs monitoring using differential microwave capacitive resonant transducer and conductive PEDOT: PSS-MWCNTs nanocomposite film for environmental applications. IEEE Trans. Nanotechnol. 2018. [CrossRef]

21. Karuppuswami, S.; Matta, L.L.; Alocilja, E.C.; Chahal, P. A Wireless RFID Compatible Sensor Tag Using Gold Nanoparticle Markers for Pathogen Detection in the Liquid Food Supply Chain. IEEE Sens. Lett. 2018, 2, 1-4. [CrossRef] 
22. Bogner, A.; Steiner, C.; Walter, S.; Kita, J.; Hagen, G.; Moos, R. Planar Microstrip Ring Resonators for Microwave-Based Gas Sensing: Design Aspects and Initial Transducers for Humidity and Ammonia Sensing. Sensors 2017, 17, 2422. [CrossRef] [PubMed]

23. Salim, A.; Memon, M.U.; Lim, S. Simultaneous Detection of Two Chemicals Using a TE20-Mode Substrate-Integrated Waveguide Resonator. Sensors 2018, 18, 811. [CrossRef] [PubMed]

24. Hasan, M.M.; Faruque, M.R.I.; Islam, S.S.; Islam, M.T. A new compact double-negative miniaturized metamaterial for wideband operation. Materials 2016, 9, 830. [CrossRef] [PubMed]

2018 by the authors. Licensee MDPI, Basel, Switzerland. This article is an open access article distributed under the terms and conditions of the Creative Commons Attribution (CC BY) license (http:/ / creativecommons.org/licenses/by/4.0/). 